\title{
Resiliencia y felicidad: el impacto de hechos vitales negativos y positivos en adolescentes
}

\author{
Norma Ivonne González-Arratia López-Fuentes ${ }^{\star}$, José Luis Valdez Medina ${ }^{\star \star}$
}

* Doctora en Investigación Psicológica. Miembro del Sistema Nacional de Investigadores Nivel II. Profesora investigadora de tiempo completo, Facultad de Ciencias de la Conducta, Universidad Autónoma del Estado de México, UAEM.

Correo electrónico:

nigalf@yahoo.com.mx

** Doctor en Psicología Social. Miembro del Sistema Nacional de Investigadores Nivel II. Profesor investigador de tiempo completo, Facultad de Ciencias de la Conducta, Universidad Autónoma del Estado de México, Uaem. Correo electrónico: ochocedros@live.com.mx

Recibido: 12 de agosto del 2013 Aprobado: 21 de octubre del 2013

Cómo citar este artículo: González-Arratia López-Fuentes, N. I. y Valdez Medina, J. L. (2013). Resiliencia y felicidad: el impacto de hechos vitales negativos y positivos en adolescentes. Pensando Psicología, 9(16), 43-53.

\begin{abstract}
Resumen
Se llevó a cabo un estudio retrospectivo con adolescentes para identificar diferencias de resiliencia entre hombres y mujeres y su relación con la felicidad después de vivenciar hechos tanto positivos como negativos del pasado reciente y que son referidos por los participantes. Cien adolescentes, hombres y mujeres, entre 15 y 19 años de edad, participaron del estudio aplicándose el cuestionario de resiliencia para adolescentes (González-Arratia, 2011), la escala de felicidad (Alarcón, 2006) y la escala de Corsini (2004). Los resultados indican que no hay diferencias significativas entre hombres y mujeres. Se reporta relación positiva entre las variables resiliencia y felicidad. Los hechos negativos tienen un efecto sobre la resiliencia, mientras que los positivos la favorecen. Se concluye que se requiere continuar analizando estas variables con el fin de obtener respuestas consistentes.
\end{abstract}

Palabras clave: adolescentes, felicidad, hechos negativos, positivos, resiliencia.

\section{Resilience and Happiness: Impact of Negative and Positive Events on Adolescents}

\section{Abstract}

A retrospective study was carried out among adolescents to identify differences in resilience among men and women and their relationship to happiness, after having gone through both positive and negative events in the recent past and which are referred to by the participants. 100 adolescent men and women between the ages of 15 and 19 took part in the study, applying the resilience for adolescents questionnaire (González-Arratia, 2011), the happiness scale (Alarcón, 2006) and the Corsini scale (2004). The results show that there are no significant differences between men and women. A positive relationship is reported between the variables for resilience and happiness. Negative effects have an effect on resilience whereas positive ones favorite. The conclusion is that further analysis of these variables is needed in order to obtain consistent responses.

Keywords: teens, happiness, negative events, positive events, resilience.

Resiliência e felicidade: $o$ impacto de fatos vitais negativos e positivos em adolescentes

Resumo

Realizou-se um estudo retrospectivo com adolescentes para identificar diferenças de resiliências entre homens e mulheres e sua relação com a felicidade depois de vivenciar fatos tanto positivos quanto negativos do passado recente e que são referidos pelos participantes. Cem adolescentes, homens e mulheres, entre 15 e 19 anos de idade, participaram do estudo no qual foi aplicado o questionário de resiliência para adolescentes (González-Arratia, 2011), a escala de felicidade (Alarcón, 2006) e a escala de Corsini (2004). Os resultados indicam que não há diferenças significativas entre homens e mulheres. Constatase relação positiva entre as variáveis resiliência e felicidade. Os fatos negativos têm um efeito sobre a resiliência, enquanto os positivos a favorecem. Conclui-se que se requer continuar analisando essas variáveis a fim de obter respostas consistentes.

Palavras-chave: adolescentes, felicidade, fatos negativos, positivos, resiliência. 
En el campo de la psicología positiva, la investigación en resiliencia se hace cada vez más relevante debido a que los individuos pueden desarrollarse psicológicamente sanos a pesar de la adversidad y el riesgo, además de que es un ejemplo de los fenómenos psicológicos que contribuyen al bienestar humano. Para abordar el tema se tomará como punto de partida la definición respecto a resiliencia, para posteriormente revisar el concepto de felicidad.

\section{Resiliencia}

En primer lugar, podemos señalar que la resiliencia tiene una larga historia de evolución, no sólo biológica sino también sociocultural, que ha ido equipando a los seres humanos de herramientas para incrementar la capacidad adaptativa de su funcionamiento; de ahí la importancia de su investigación. Es, pues, una disposición clave para el funcionamiento óptimo de los individuos. El concepto de resiliencia resulta fácil de entender, pero complejo de definir, ya que asumimos que es multidimensional, lo cual implica factores tanto individuales, familiares, socioculturales, como de interacción de la persona y su entorno.

En la revisión bibliográfica al respecto, se observa que el término resiliencia tiene múltiples conceptualizaciones, y varios autores ofrecen perspectivas críticas que proponen afinar el concepto y la manera como se investiga. Ungar (2005) nos invita a analizar cuidadosamente el concepto, y señala que se usa para referirse tanto a una cualidad como a un rasgo, un proceso y un resultado, lo que hace que el término resulte ambiguo. Sin embargo, la mayoría de las definiciones coinciden en definirla como aquella cualidad de las personas para resistir y rehacerse ante situaciones traumáticas o de pérdida. Masten (2011) señala que no es un fenómeno directamente observable, sino que más bien es un concepto inferencial, por lo que llegamos a la conclusión de que una persona es resiliente si funciona bien tras haber sufrido experiencias traumáticas o estresantes.

La resiliencia es, entonces, un constructo inferido que requiere el cumplimiento de tres características esenciales: la primera de ellas consiste en determinar la existencia de una amenaza al desarrollo; la segunda consiste en que la persona adquiera, a partir de sus interacciones psicológicas, la disposición para la resiliencia, esto es, la tendencia a la superación y posterior adaptabilidad ante las adversidades, y por último, es necesario demostrar la competencia en una tarea del desarrollo de acuerdo con la edad y con la cultura (Gaxiola, Frías, Hurtado, Salcido y Figueroa, 2011).

También se piensa que la resiliencia es la aptitud para soportar las crisis, el estrés y las adversidades en forma positiva, recobrando la fortaleza o resistencia para salir airoso de la situación. Implica enfrentar los problemas que producen estrés, advirtiendo posibilidades de superarlos y, sobre todo, de recuperarse y fortalecerse con la experiencia. Desde esta concepción, entonces, los problemas y las situaciones constantes de crisis pueden ser enfrentados y superados buscando las particularidades positivas que llevan a mejorar la situación futura. Por tanto, la capacidad de resiliencia consiste en recuperarse de los conflictos no únicamente dejando que sólo desaparezca la crisis, sino impulsando cambios y fortaleciendo las defensas ante la reaparición de estos.

Por otro lado, el papel de la interacción entre factores de riesgo y de protección ha sido señalada en la investigación en resiliencia (González Arratia, 2011), lo cual sugiere retomar los estudios de Grotberg (1995), en el sentido de que ningún factor en particular y por sí solo promueve la resiliencia, lo que justifica la visión multinivel en el estudio del constructo y así conocer con mayor precisión los factores involucrados en el comportamiento resiliente.

De entre los distintos estudios empíricos, la presente investigación aborda el constructo resiliencia desde el modelo de González Arratia (2011) que indica que la resiliencia se sustenta en la interacción entre el individuo y el entorno, por lo que se entiende que la resiliencia es el resultado de la combinación o la interacción entre los atributos del individuo (internos) y su ambiente familiar, social y cultural (externos) que lo posibilitan para superar el riesgo y la adversidad de forma constructiva (González Arratia, 2011). Es importante mencionar que esta definición ha sido el eje central para el desarrollo de toda la investigación.

Existe un punto motivo de análisis: el establecimiento de semejanzas y diferencias entre hombres y mujeres respecto al nivel de resiliencia. Mientras que algunos autores reportan que existen diferencias entre hombres y mujeres (Consedine, Magai y Krivoshekova, 2005; Nygren, Jonsen, Gustafson, Norberg y Lundman, 2005), hay quienes no encuentran diferencia alguna (Keneally, 1993). También se ha encontrado que ambos presentan la misma frecuencia de conductas resilientes: las niñas tienden a contar con habilidades interpersonales y fortaleza interna, en tanto los niños tienden a ser más pragmáticos, por lo que se hace necesario continuar ana- 
lizando esta variable desde una perspectiva empírica con el fin de identificar su influencia. Si bien no existe un consenso entre los autores respecto a posibles diferencias, en este estudio se plantea que los hombres y las mujeres difieren en cuanto a sus características resilientes.

\section{Felicidad}

Estudios recientes han dejado claro que la felicidad está estrechamente relacionada con la resiliencia (Salgado, 2009); además, es un factor protector que promueve el funcionamiento positivo en contextos de inestabilidad (Veronese, Castigliani, Tombolani y Said, 2012). La mayoría de las lenguas occidentales utilizan las siguientes palabras para designar el concepto de felicidad: happiness proviene del inglés happ, que significa ocasión, fortuna; de aquí evolucionará también el verbo "to happen" (suceder, ocurrir). El término francés bonheur, procede de bon (bueno) y heur (suerte o fortuna). En italiano, español, portugués y catalán, felicità, felicidad, felicidade y felicitat derivan del término en latín felix, que a veces significa suerte y, otras, destino. Y, curiosamente, aunque es en los albores de la humanidad, cuando se empieza a relacionar la felicidad con el azar, a partir de Aristóteles y hasta la Edad Media el concepto de felicidad se podía resumir en dos corrientes: la que consideraba la satisfacción de los placeres como la felicidad, y la que postulaba que para lograr la felicidad debería incluirse la virtud y la sabiduría.

Generalmente la felicidad es definida como un estado de bienestar subjetivo, o bien como sentir satisfacción con la vida, o como la evaluación que las personas hacen de sus vidas (Diener, 2009). Al respecto, Diener (2006) también menciona la dificultad que existe para pensar una definición de felicidad, la cual ha sido utilizada para referirse a distintas ideas, tales como: tener un humor positivo, una evaluación global de la satisfacción con la vida, la vivencia de una buena vida, o remiten a las causas que hacen sentir felices a las personas o a diversas interpretaciones que dependen de los contextos en los cuales se desarrollan. Esta dificultad ha generado que algunos académicos eviten la utilización de este concepto y prefieran utilizar términos específicos de los diferentes aspectos del bienestar. No obstante, también hay quienes prefieren usarlo teniendo en cuenta su valor histórico y su popularidad. En general existe un consenso en cuanto a las dimensiones que conforman la felicidad: la satisfacción vital, el afecto positivo y el afecto negativo.
Se considera que la felicidad tiene una dimensión básica y general que es subjetiva, que a su vez tiene dos componentes: uno centrado en los aspectos afectivosemocionales referidos a la frecuencia y la intensidad de emociones positivas y negativas, con una preponderancia de las primeras, y otro centrado en los aspectos cognitivos referidos a la evaluación de la satisfacción que hace la persona de su propia vida (Andrews y Withey, 1976; Diener, Suh, Lucas y Smith, 1999; Cuadra y Florenzano, 2003).

Investigaciones recientes apuntan a examinar la felicidad o el bienestar subjetivo (Diener, 2009), y revelan que la felicidad es igualmente accesible para jóvenes y menos jóvenes, varones y mujeres. El conocimiento de la edad, el sexo, la etnicidad y el nivel de ingresos, ofrecen pocas indicaciones sobre la felicidad. La felicidad depende poco de elementos externos. Los mejores indicios provienen de los rasgos de personalidad. Se han identificado tres rasgos de la gente feliz: el primero es la autoestima; el segundo, la persona típicamente feliz percibe estar en posesión de su vida (Larson, 1989), y tercero, es optimista (Diener, 2009; Toribio, González Arratia, Valdez, González y Oudhof, 2012).

Por otro lado, investigaciones actuales han conectado la resiliencia con la felicidad (Salgado, 2009) indicando que existe una relación altamente significativa entre estas variables, resaltando la importancia que tienen en la salud emocional de la persona. Respecto a la relación entre felicidad y género, Myers y Diener (1995) hallaron que los hombres y las mujeres se consideran muy felices y satisfechos en igual número.

Según Michalos (2007), las percepciones, los pensamientos y las acciones de las personas tienen un impacto en sus condiciones de vida y en la de los demás. Al respecto se ha planteado que los adolescentes resilientes experimentan un mayor número de emociones positivas, lo cual aumenta su bienestar subjetivo (Omar, Paris, Uribe, Ameida y Aguilar, 2011). Asimismo, se ha observado que las personas infelices reportan más eventos negativos o de cambios en la vida con efectos adversos (accidentes y pérdida de trabajo), así como menos eventos positivos (Palomar y Victorio, 2012; Veenhoven, 2004).

Además, la evidencia ha demostrado relaciones positivas entre resiliencia y afectos positivos, y confianza para afrontar las dificultades y apoyo social percibido (Omar, 2008). Por su parte, Helgeson, Reynolds y Tomich (2006) encontraron que un mayor crecimiento postraumático se asociaba con una menor depresión y un mayor bienestar psicológico, aunque no había aso- 
ciación con medidas de balanza de afecto que incluyen indicadores de emociones positivas y negativas simultáneamente, lo que sugiere que el crecimiento coexiste con ambos tipos de emociones.

Se ha observado, como se anotó, que las personas infelices reportan más eventos negativos o de cambios en la vida con efectos adversos, tales como accidentes y pérdida de trabajo, así como menos eventos positivos, tales como enamorarse, conseguir alguna meta (Veenhoven, 2004).

Así, la relación entre ambas variables queda expresada con la siguiente hipótesis: la felicidad está positivamente relacionada con la resiliencia. Después de haber revisado brevemente ambos conceptos, es necesario pasar ahora a los que se refieren los hechos negativos y positivos.

\section{Impacto de hechos negativos}

El desarrollo de nuevas posibilidades y cambio de probabilidades en la vida lo producen tanto hechos positivos como negativos extremos. Respecto a los hechos negativos, estudios como los de Baumeister, Bratslavsky, Finkenauer y Vohs (2001) señalan que estos tienen un impacto mayor y más duradero en las personas que los hechos positivos, a pesar de que estos son más frecuentes. Una de las explicaciones es que los hechos negativos impactan más en la afectividad y el pensamiento de las personas, ya que generalmente son contrarios a sus expectativas e intensiones, además de ser menos frecuentes.

Asimismo, se ha visto que se reacciona más fuertemente utilizando más recursos emocionales, cognitivos y fisiológicos en respuesta a eventos negativos. Se piensa más en ellos, se busca más en explicarlos y afrontarlos que los hechos positivos.

Baumeister et al. (2001) consideran que los individuos tienen la predisposición a responder más rápida e intensamente a hechos negativos puesto que se tiene una base evolutiva. El costo de perder oportunidades o recursos es menor que el de no percibir amenazas, por lo que en el desarrollo de la especie se ha seleccionado como un rasgo adaptativo esta predisposición a lo negativo. Pero también se ha reportado que luego de una gran reacción a los eventos negativos, por lo general se minimiza su influencia o se re-evalúan hasta neutralizar el impacto que generaron. Bilbao (2009) halló que los hechos negativos se asocian con una menor satisfacción con la vida, menor felicidad y bienestar eudamónico.
Un estudio sobre la influencia de los hechos vitales mostró que los hechos negativos ocurridos hace tres meses o menos disminuyen la satisfacción con la vida; sin embargo, a los seis meses esta relación no es significativa (Páez, Bilbao y Javaloy, 2009).

Bonanno (2004) propone cuatro patrones diferentes o trayectorias ante la exposición a un contexto adverso: disfunción crónica, recuperación, resiliencia y disfunción retardada. Las personas resilientes serían aquellas que, a pesar de enfrentarse a un acontecimiento traumático, son capaces de mantener un funcionamiento normal a lo largo del tiempo, mientras que las personas que se recuperan manifestarían síntomas psicopatológicos, al menos durante algunos meses, y luego se recuperarían y serían diversos los factores personales y contextuales, como por ejemplo el apoyo social, los que determinarían las diferentes trayectorias (Ruiz y López, 2012).

Los hechos traumáticos pueden producir una visión negativa de sí mismo y del mundo, pero también conllevan efectos positivos en el ámbito personal, tales como: a) crecimiento personal, aprender sobre las capacidades, habilidades y resistencia personal; b) aumentar la sabiduría y el conocimiento; c) mejorar el conocimiento sobre sí mismo y los demás; d) apreciar lo que se tiene y aprender las prioridades importantes en la vida, y e) crecimiento y desarrollo espiritual.

Asimismo, si un trauma o estrés pueden empeorar la visión del mundo y de los otros, también puede tener efectos positivos en las relaciones con los demás, como pueden ser los siguientes: a) reunir y acercar a la familia, b) cohesionar a la comunidad, haciéndolos sentirse más cerca; c) provocar una orientación más prosocial: ser más tolerante y compasivo con los otros, así como valorar el apoyo que estos ofrecen, y d) pensar que los otros pueden beneficiarse de su experiencia.

\section{Impacto de hechos positivos}

En cuanto a los hechos vitales positivos ocurridos hace tres meses o menos, se ha encontrado que estos tienen una influencia significativa en la satisfacción con la vida; los hechos negativos, a su vez, disminuyen la satisfacción con la vida en el mismo periodo (Such, Diener y Fujita, 1996).

Fredrickson (2009) señala que en el caso de eventos traumáticos, las emociones positivas son una base importante para promover un crecimiento positivo. Un 
creciente número de estudios empíricos revela que los sobrevivientes de trauma experimentan muchos cambios psicológicos positivos posteriormente. Además, las personas informan de resultados positivos después de haber vivido situaciones extremadamente estresantes, ya sea como resultado directo del evento o como un tipo de aprendizaje que se produjo por medio de sus esfuerzos para hacer frente a los hechos (Park, 1998).

Así, se ha encontrado que después de vivir experiencias de este tipo, la mayoría de las personas encuentran aspectos positivos en ellas (Páez et al., 2009; Páez, Campos y Bilbao, 2008). Al respecto se ha observado que al re-evaluar o reintepretar positivamente un hecho y sus efectos, el crecimiento postraumático se ha asociado con la experiencia de emociones positivas, y mediaba con la resiliencia y el crecimiento postraumático unos meses después (Zoellner y Maercker, 2006). Según el estudio de Helgeson, Reynolds y Tomich (2006), las personas dicen estar mejor ahora que antes del hecho negativo, porque revisan a la baja sus debilidades y características previas, es decir, se ven más seguros y fuertes ahora porque recuerdan equivocadamente que antes eran más débiles (Tennen y Affleck, 2005).

También existe evidencia de que las personas experimentan una variedad amplia de emociones y cogniciones positivas, además de los beneficios sociales percibidos a menudo después de las adversidades - como por ejemplo sentir mayor unión familiar, un aumento del apoyo social, de la empatía y más comportamientos prosociales-, ya que, en el caso de los acontecimientos sociales traumáticos, las personas también pueden experimentar un aumento agudo de la cohesión de su comunidad (Vázquez y Hervás, 2008).

Otro elemento que explicaría el bienestar sería que no sólo los hechos positivos refuerzan la autoestima, las relaciones positivas con otros y el sentido de la vida, sino que incluso los hechos negativos extremos provocan crecimiento personal, sugiriendo que los hechos estresantes servirían de catalizador o aliciente, como confirma la investigación sobre crecimiento postraumático.

En este contexto, y desde una mirada ecosistémica, la presente investigación es un estudio retrospectivo transversal en el que se desea responder varias preguntas tales como: qué relación hay entre resiliencia y felicidad en adolescentes después de haber experimentado hechos considerados por el propio individuo como positivos y negativos, y saber si difieren los hombres y las mujeres respecto a estas variables. Este trabajo se apoya en el supuesto de que el efecto que tienen diferentes eventos no es el mismo si este es positivo o negativo, y se realiza en el campo de la psicología positiva, el cual aún es incipiente en México, por lo que se justifica su estudio. Sin duda alguna, son cuestiones complejas de responder con el fin de aportar datos que nos den respuestas consistentes, por lo que se considera conveniente realizar un estudio sobre cómo interactúan estas variables.

\section{Método}

\section{Participantes}

En este estudio participaron adolescentes, para lo cual se trabajó con una muestra no probabilística intencional compuesta por 100 adolescentes igualmente representados entre 15 a 19 años de edad $(X=15,40, D S=68), y$ que en el momento del estudio cursaban el nivel bachillerato, todos de la ciudad de Toluca, Estado de México.

\section{Instrumentos}

Se aplicaron los tres instrumentos que se describen en los siguientes apartados.

\section{Cuestionario de resiliencia para niños y adolescentes de González Arratia (2011)}

Instrumento de autoinforme previamente desarrollado en México para niños y adolescentes, que mide factores específicos de la resiliencia basada en los postulados de Grotberg (2006), organizados en tres categorías: yo tengo (apoyo), yo soy y estoy (atañe al desarrollo de fortaleza psíquica), yo puedo (remite a la adquisición de habilidades interpersonales). Consta de 32 reactivos con un formato de respuesta tipo Likert de 5 puntos: el valor (1) indica "Nunca" y el (5) "Siempre". Se llevó a cabo un análisis factorial exploratorio con el método de componentes principales, desde el supuesto de que son factores no correlacionados o independientes. Para tal fin el método rotación ortogonal (varimax) se considera el más adecuado (Landero y González, 2006). Se cumple el criterio de Kaiser (кмо $=0,9, p=0,001)$ y la prueba de esfericidad de Barttett (Chi-cuadrado aproximado = 1673,$3 ; \mathrm{p}=0,000$ ) por lo que resulta adecuado realizar el análisis factorial. A partir de lo anterior, se obtuvo una varianza total explicada con los 32 reactivos de $40,332 \%$. El primer factor explica el $14,83 \%$ de la varianza; en el caso del factor 2 fue de $13,51 \%$ y el factor 3 con una varianza de $11,98 \%$. Se obtuvo una consistencia interna de Alfa de Cronbach alta (total $=0,9192$ ). En otro estudio se llevó a cabo análisis factorial confirmatorio en el que 
se comprueba la tridimensionalidad del instrumento de resiliencia (González Arratia y Valdez, 2012):

1. Factores protectores internos. Mide habilidades para la solución de problemas $(\alpha=0,8050$ con 14 reactivos).

2. Factores protectores externos. Evalúa la posibilidad de contar con apoyo de la familia o personas significativas para el individuo ( $\alpha=0,7370$ con 11 reactivos).

3. Empatía. Se refiere a comportamiento altruista y prosocial ( $\alpha=0,7800$, con 7 reactivos).

La calificación del cuestionario está dada por la suma del puntaje total. Los valores altos indican mayor resiliencia, es decir que los individuos que presentan alto puntaje en los tres factores que integran el instrumento poseen habilidades para la solución de problemas, cuentan con redes de apoyo para la superación de los problemas, así como comportamiento altruista y prosocial, los cuales son componentes indispensables para la resiliencia (González Arratia, 2011).

\section{Escala de hechos negativos-positivos de Corsini}

Se aplicó la escala modificada en España por Páez. Esta versión consta de 12 ítems: seis de vulnerabilidad o disminución de creencias básicas positivas (negativos impares) y seis de invulnerabilidad o aumento de creencias básicas positivas (positivos pares). Dos ítems representan cada una de las dimensiones siguientes: visión del yo digno y de respeto; aumento de la motivación y eficacia; visión del mundo social benevolente y de los otros; integración social: visión del mundo benevolente, y sentido del mundo. Las respuestas puntúan en una escala de tipo Likert, con rango (1) ("Totalmente falso") a (7) (“Totalmente verdadero"), y tiene una buena fiabilidad, presentando los ítems de vulnerabilidad un Alfa de Cronbach de 0,86 y los de invulnerabilidad, de 0,87, según lo reportado por Bilbao, Páez, Da Costa y Martínez (2013).

Evalúa hechos impactantes del pasado reciente (en esta investigación se consideró de 6 meses), para lo cual se le solicita al participante que piense en el momento en que ocurrió el hecho y responda en qué medida tuvo los efectos que se le preguntan. El autor reporta que la suma de los doce ítems es de 53,7, con una media general de $M=4,4$. Las puntuaciones inferiores a 3 sugieren que la experiencia socavó las creencias básicas (Corsini,
2004). Con el objetivo de conocer los hechos positivos se aplicó el mismo instrumento adaptándolo a hechos positivos. Para la presente investigación se obtuvo un alfa de 0,892 y 0,920 para hechos negativos y positivos, respectivamente. Es importante indicar que se incluyó una pregunta en la que se solicitaba al individuo que explicara brevemente el suceso tanto positivo como negativo.

\section{Escala de felicidad de Alarcón (2006)}

Consta de 27 ítems construidos en escala Likert con cinco alternativas de respuesta. El extremo positivo tiene un valor de 5 puntos, y el extremo negativo, 1 punto. De acuerdo con el puntaje total, el autor los divide en cinco niveles de felicidad: de 27-87 puntos, muy baja felicidad; 88-95: baja; 96-110: media; 111-118: alta; 119 135: muy alta. Con 4 dimensiones: sentido positivo de la vida, satisfacción con la vida, realización personal y alegría de vivir, el autor reporta un coeficiente de confiabilidad total alfa de 0,916. La escala fue previamente validada en muestras de adolescentes mexicanos, para lo cual se llevó a cabo un análisis factorial exploratorio con rotación ortogonal en el que se extrajeron 4 factores con un porcentaje total de varianza explicada de 50,32\%. El primer factor se encuentra compuesto por 10 reactivos y se denomina sentido positivo de la vida, que explica el $18,12 \%$ de la varianza total. El segundo factor es satisfacción con la vida, integrado por 6 ítems y una varianza de $11,22 \%$. El factor 3 es realización personal con 5 ítems. Por último, el factor 4 se denominó alegría de vivir, con 5 ítems y $10,27 \%$ de varianza, conservándose 26 reactivos de los 27 originales, y una confiabilidad Alfa de Cronbach de 0,917 (Toribio et al., 2012). La versión con 26 reactivos fue aplicada a la muestra bajo estudio.

\section{Procedimiento}

Todos los participantes completaron las tres escalas en una sesión y en un tiempo aproximado de 45 minutos en los respectivos salones de clase, previo permiso de la Dirección y en horarios académicos. La participación fue voluntaria, anónima y confidencial, y se atendieron las dudas que surgieron en el momento de la aplicación.

\section{Análisis de resultados}

Se realizaron análisis descriptivos para obtener media y desviación estándar, se aplicó la prueba de normalidad de Kolmogorov-Smirnov. A partir del puntaje total 
de la escala de resiliencia se establecieron puntos de corte utilizando para ello percentiles (25-75). La prueba $t$ de Student se usó para determinar diferencias según las variables grupo (resiliente-no resiliente) y sexo (hombre-mujer). También se llevó a cabo un análisis con la prueba Chi cuadrado, y análisis de correlación de Pearson para establecer la relación entre las variables resiliencia y felicidad, considerando un nivel de significancia de $\mathrm{p} \leq 0,05$. Los análisis estadísticos se llevaron a cabo en el programa sPss versión 20 .

\section{Resultados}

Respecto a la pregunta sobre cuál había sido el suceso que consideró como hecho negativo, se obtuvo una amplia gama de eventos, los cuales se agruparon en categorías: familiar, social, escolar y sí mismo. En relación con la primera categoría, indicaron: dificultades con los padres, separaciones o divorcios y muerte de un ser querido, además de problemas con la pareja y los hijos. Un segundo conjunto de eventos se agrupó en la dimensión social, que incluye los hechos negativos que con mayor frecuencia reportaron los adolescentes, y son: problemas con los amigos y ruptura de noviazgo; le siguen los eventos de la dimensión escolar que tiene que ver con situaciones como los exámenes, ser dado de baja, bajas calificaciones y falta de cumplimiento con trabajos y tareas. Finalmente, en la dimensión sí mismo, se incluyeron hechos tales como una enfermedad grave, haber sido víctima de un abuso y robo.

En el caso de los hechos positivos también se clasificaron en estas dimensiones, y los que más frecuentemente fueron reportados para la dimensión familia fueron: formar una familia y casarse. En la dimensión social: tener novio(a); en la dimensión escolar indicaron aprobar una materia, y en el caso de la dimensión sí mismo, se incluyeron: tener dinero, salir de un problema y ayudar a un conocido a salir de un problema.
En lo relativo a los análisis estadísticos de tipo descriptivo, se inició con un análisis con el objetivo de comprobar la distribución de los datos considerando el puntaje total de cada escala, en donde se observó que se ajustan a la curva normal; por tanto se rechaza la hipótesis nula (resiliencia Zk-s $=0,891, \mathrm{p}=0,405$; felicidad $\mathrm{Zk}-\mathrm{s}=0,721, \mathrm{p}=0,676$; hechos $\mathrm{Zk}-\mathrm{s}=1,35, \mathrm{p}=0,051$ ), por lo que se considera que se da cumplimiento del supuesto de normalidad (Landero y González, 2006), de tal forma que se decidió proceder al cálculo de análisis de diferencias.

Con el objetivo de diferenciar a los individuos resilientes de los no resilientes, se procedió de la siguiente manera: en primer lugar, se obtuvieron las puntuaciones de los niveles de resiliencia para esta muestra; se establecieron para ello los percentiles 25 (grupo baja resiliencia, $\mathrm{n}=34$, de los cuales son 17 hombres y 17 mujeres) y 75 (grupo alta resiliencia, $\mathrm{n}=25,15$ son hombres y 10 mujeres); el resto $(\mathrm{n}=41)$ se consideró con puntajes de resiliencia moderada.

La comparación entre resilientes y no resilientes se hizo con la prueba t de Student, que indica que sí hay diferencia entre el grupo de alta y baja resiliencia $(t=$ $-12,56, \mathrm{p}=0,001$; Media grupo alta resiliencia $=1,49$, $\mathrm{DS}=11,46$; Media grupo baja resiliencia $=1,26$, DS $=$ $5,70)$. Se encontró un porcentaje mayor de adolescentes que presentan baja resiliencia (34\%), a los cuales se les considera como no resilientes; en el caso de los que se consideran resilientes, corresponde al $25 \%$ de la muestra total. Con base en estos criterios, hay 34 participantes clasificados como no resilientes y 25 clasificados como resilentes, y el resto (41) no pertenecía a ninguno de estos grupos.

A partir de este criterio, posteriormente se llevó a cabo un análisis de $t$ de Student para observar si había diferencias entre el grupo de individuos resilientes y no resilientes, por lo que se hizo un análisis de diferencias con la prueba t de Student, asumiendo que las varianzas son iguales (prueba de Levene para igualdad de la varianza, $95 \%$ de confianza) en la que se encontraron

Tabla 1. Diferencias en grupos Alta y Baja resiliencia, t de Student

\begin{tabular}{|c|c|c|c|c|c|c|c|}
\hline & Dimensión & $\mathrm{p}$ & $\mathbf{t}$ & $\begin{array}{c}\text { Media } \\
\text { Grupo Alta resiliencia } \\
n=25\end{array}$ & Ds & $\begin{array}{c}\text { Media } \\
\text { Grupo Baja resiliencia } \\
\mathbf{n}=34\end{array}$ & Ds \\
\hline \multirow{5}{*}{ Felicidad } & Sentido positivo de la vida & 0,298 & 1,04 & 2,56 & 0,36 & 2,63 & 0,33 \\
\hline & Realización personal & 0,001 & $-3,38$ & 3,36 & 0,44 & 3,08 & 0,38 \\
\hline & Satisfacción con la vida & 0,001 & $-3,94$ & 4,24 & 0,62 & 3,73 & 0,66 \\
\hline & Alegría de vivir & 0,103 & 1,64 & 1,52 & 0,76 & 1,75 & 0,64 \\
\hline & Felicidad total & 0,001 & $-3,94$ & 84,01 & 7,68 & 78,72 & 5,60 \\
\hline
\end{tabular}


Tabla 2. Diferencias en hombres y mujeres para la variable atributiva sexo, $\mathrm{t}$ de Student

\begin{tabular}{|c|c|c|c|c|c|c|c|}
\hline & Dimensión & p & $t$ & $\begin{array}{c}\text { Media } \\
\text { hombres } \\
\mathbf{n}=50\end{array}$ & Ds & $\begin{array}{c}\text { Media } \\
\text { mujeres } \\
\mathrm{n}=50\end{array}$ & Ds \\
\hline \multirow{4}{*}{ Resiliencia } & Factor protector interno & 0,21 & 1,25 & 4,34 & 0,44 & 4,21 & 0,33 \\
\hline & Factor protector externo & 0,63 & 0,47 & 4,50 & 0,46 & 4,54 & 0,41 \\
\hline & Empatía & 0,60 & 0,51 & 4,04 & 0,72 & 4,11 & 0,65 \\
\hline & Resiliencia total & 0,78 & 0,27 & 1,38 & 14,54 & 1,37 & 0,67 \\
\hline \multirow{5}{*}{ Felicidad } & Sentido positivo de la vida & 1,0 & 0,00 & 2,60 & 0,36 & 2,60 & 0,33 \\
\hline & Satisfacción con la vida & 1,0 & 0,00 & 3,22 & 0,45 & 3,22 & 0,41 \\
\hline & Realización personal & 0,49 & 0,68 & 4,03 & 0,74 & 3,94 & 0,65 \\
\hline & Alegría de vivir & 0,89 & 0,13 & 1,62 & 0,75 & 1,64 & 0,67 \\
\hline & Felicidad total & 0,77 & 0,29 & 81,58 & 8,54 & 81,16 & 5,63 \\
\hline
\end{tabular}

Fuente: elaboración propia

diferencias estadísticamente significativas que indican que los individuos que puntúan con alta resiliencia presentan mayor realización personal y satisfacción con la vida y el total de felicidad, en comparación con el grupo considerado con baja resiliencia. Para las dimensiones sentido positivo de la vida y alegría de vivir no se encontraron diferencias significativas (ver tabla 1).

Según la variable atributiva sexo, no se encontraron diferencias significativas; sin embargo, las medias indican que las mujeres puntúan ligeramente más alto en la dimensión alegría de vivir de la escala de felicidad, mientras que los hombres presentan mayor factor protector interno de la escala de resiliencia. La edad sólo fue significativa en el factor protector interno de resiliencia a favor de los individuos entre 17 a 19 años (Media $=4,41, \mathrm{DS}=0,43, \mathrm{p}=0,036)$ y menor para los que tienen entre 15 y 16 años (Media $=4,21$, DS =,51); en el resto de las dimensiones no hay diferencias.

Respecto a la relación entre resiliencia y felicidad, se llevó a cabo un análisis de correlación de Pearson entre las escalas, considerando una $\mathrm{p} \leq 0,05$, lo cual revela que las dimensiones de resiliencia y felicidad se asocian en el sentido esperado, es decir, correlacionan significativamente, aunque son consideradas de moderadas a bajas. Para cada uno de los factores de resiliencia se halló que a mayor factor protector interno, mayor satisfacción y alegría de vivir. El factor protector externo se asocia positivamente con satisfacción y alegría de vivir. El factor 3, que es empatía, se asocia con las mismas dimensiones, satisfacción y alegría de vivir. El total de resiliencia presentó correlación positiva con el total de la variable felicidad, la cual resultó significativa, y de acuerdo con los criterios de Levin (1979) este coeficiente de correlación se puede interpretar como correlación positiva de débil a moderada. El resto de las dimensiones de la escala de felicidad no correlacionó significativamente (tabla 3).
El análisis de $\mathrm{Chi}^{2}$ indica que los individuos con baja resiliencia presentan muy baja felicidad $\left(\mathrm{Chi}^{2}=\right.$ $17,19, \mathrm{p} \leq, 002)$.

Tabla 3. Resiliencia y felicidad. Correlación de Pearson

\begin{tabular}{lccccc}
\hline & $\mathbf{1}$ & $\mathbf{2}$ & $\mathbf{3}$ & $\mathbf{4}$ & $\mathbf{5}$ \\
\hline $\begin{array}{l}\text { Factor protector } \\
\text { interno }\end{array}$ & - & $0,403^{* *}$ & - & $0,243^{*}$ & - \\
$\begin{array}{l}\text { Factor protector } \\
\text { externo }\end{array}$ & - & $0,559^{* *}$ & - & $0,310^{*}$ & - \\
$\begin{array}{l}\text { Factor empatía } \\
\text { Total resiliencia }\end{array}$ & - & $0,465^{* *}$ & - & $0,215^{*}$ & - \\
\hline
\end{tabular}

1. Sentido positivo de la vida. 2. Satisfacción con la vida. 3. Realización personal. 4. Alegría de vivir. 5. Total felicidad. ${ }^{*} \mathrm{p}=0,05{ }^{\star *} \mathrm{p}=0,01$

Al recordar un hecho de afectividad negativa, aplicando la escala de Corsini (2004), los resultados indican una experiencia de afectividad neutra (Media = $3,77$, Ds $=1,34$, mínimo $=1$, máximo $=6,7)$. En el caso de los hechos positivos, la experiencia que se recordó tuvo un ligero impacto en sus creencias básicas (Media $=4,34, \mathrm{DS}=1,79$, mínimo $=1$ máximo $=6,50)$.

Se observó un efecto interesante que muestra que cuando los hechos negativos hacen que se pierda la confianza en la gente $\left(\mathrm{Chi}^{2}=21,61, \mathrm{p} \leq 0,042\right)$ y la persona se siente desmoralizada $\left(\mathrm{Chi}^{2}=21,87, \mathrm{p} \leq 0,039\right)$, hay menor resiliencia. En cuanto a los hechos positivos, la respuesta de sentirse capaz se asocia con mayor resiliencia $\left(\mathrm{Chi}^{2}=30,63, \mathrm{p} \leq 0,006\right)$.

\section{Discusión}

Sobre la base de los resultados se encontró que en mayor medida los adolescentes presentaron un nivel bajo de resiliencia, lo cual es un posible indicador de un déficit de la variable estudiada y se consideran que re- 
quieren mayores habilidades cognitivas, interpersonales y emocionales con el fin de sobreponerse a alguna situación de adversidad a las que puedan estar expuestos (González Arratia, 2011).

El 25\% de la muestra total fue clasificado en el grupo con alta resiliencia, lo cual podría ser un indicador que favorece a los individuos para desenvolverse en el ámbito social y relacionarse con estilos de afrontamiento directo, autoestima y locus de control interno, características que están estrechamente relacionadas con la resiliencia (González Arratia, 2011). En cuanto a los adolescentes que obtuvieron puntajes medios en resiliencia, no manifiestan tener una clara necesidad de aprender autocontrol, pero se beneficiarían enormemente de esto; es decir, sería recomendable avanzar en la promoción de su nivel de resiliencia al enriquecer vínculos sociales y enseñar habilidades para la vida con el fin de que puedan fortalecer su autonomía (González Arratia y Valdez, 2012; Saavedra y Villalta, 2008; Vargas y Villavicencio, 2011).

Por otro lado, los resultados también evidencian que los adolescentes resilientes presentan en mayor medida realización personal y satisfacción con la vida y el total de felicidad, que los no resilientes. Una posible explicación al respecto es lo que señala Salgado (2006) en el sentido de que el hecho de que exista un número significativo de personas que señalan sentirse felices, a pesar de las condiciones de desarrollo que tienen, se ajusta a lo que algunos científicos denominan como los pobres felices, los cuales estarían presumiblemente satisfechos con su suerte, lo que ha sido interpretado como un estado de adaptación y de resignación adquirida.

Respecto de la variable sexo, no se encontraron diferencias significativas. Esto corrobora lo hallado en otros estudios (Rodríguez et al., 2009), por lo que se puede afirmar que la resiliencia es una capacidad que se puede desarrollar en cualquier persona sin importar su género.

Entre los hallazgos también se encontró una relación significativa entre resiliencia y felicidad, medida con la escala de Alarcón (2006). Los resultados coinciden con los de Salgado (2009), ya que él reporta una correlación similar $(r=0,44, \mathrm{p}=0,001)$ entre resiliencia y felicidad en estudiantes de 15 a 18 años de edad, y explica que una persona resiliente, que es capaz de enfrentarse a la adversidad de forma positiva, haciendo uso de diferentes recursos para superar el dolor, sufrimiento, y salir adelante de dichas circunstancias, es una persona más optimista y más feliz (González Arratia, Amado y Valdez, 2010).
En el caso de los hechos positivos-negativos yla resiliencia, estos resultados difieren a lo reportado por Páez et al. (2009), quienes consideran que es posible considerar una imagen positiva del yo en el caso de hechos negativos. Nuestros hallazgos son en el sentido opuesto, constatándose que los hechos negativos refuerzan las creencias negativas, mientras que en el caso de los positivos, los individuos refuerzan la idea de ser capaces. Lo anterior nos estaría indicando que la respuesta más esperada ante un hecho negativo es, por lo general, el dolor y el sufrimiento. Es necesario continuar investigando si en algún momento los adolescentes son capaces de ver elementos positivos ante una situación experimentada y evaluada como negativa, lo que sería una manifestación de la resiliencia de los individuos.

Lo anterior sugiere que igualmente sería interesante conocer la percepción de apoyo que tienen los adolescentes en el caso de hechos negativos, para determinar si esto favorece o no su resiliencia y felicidad autorreportada, puesto que se parte de que la felicidad es el crecimiento personal provocado por hechos de cambio vital importantes aunque sean negativos. Por tanto, es relevante dar un seguimiento a los individuos con el fin de precisar si más tarde los hechos negativos tienen consecuencias positivas y enriquecedoras en sus vidas (Csikszentmihalyi, 2007).

Un elemento central para tener en cuenta es que cuando un acontecimiento negativo o positivo altera el nivel de bienestar, determinados mecanismos de regulación se encargarían de volver al estado previo de bienestar que marca su temperamento. También se ha observado que algunas experiencias parecen reducir de forma significativa los niveles de felicidad, desafiando las capacidades adaptativas del individuo (por ejemplo, la muerte de la pareja). Y por otra parte, se ha observado de forma preliminar que ciertos entrenamientos positivos pueden elevar de forma permanente el bienestar (Seligman, Steen, Park y Peterson, 2005).

Con base en lo anterior, se considera que la implicación más importante de la actual propuesta radica en que, si bien un porcentaje de la muestra presentó puntajes altos en resiliencia y felicidad, no se sabe si estas personas no se vieron realmente afectadas en el momento en el que reportaron un hecho negativo, incluso si se recuperaron antes del momento de la medición, o si están presentes otros factores que los protegen de los efectos de una situación de riesgo, por lo que será necesario continuar probando esta hipótesis.

Además, estos resultados nos sugieren que la búsqueda de la conexión entre resiliencia y felicidad resul- 
ta compleja y presenta panoramas distintos, según la perspectiva teórica que estemos asumiendo. Por ello es necesario seguir tratando de comprender este dilema de la resiliencia, no sólo en un sentido unidireccional sino también bidireccional, aspecto que será necesario analizarlo cuidadosamente.

\section{Referencias}

Alarcón, R. (2006). Desarrollo de una escala factorial para medir felicidad. Revista Interamericana de Psicología, 40(1), 96-106.

Andrews, F. y Withey, S. (1976). Social indicators of wellbeing: American's perceptions of life quality. New York: Plenum.

Baumeister, R. F., Bratslavsky, E., Finkenauer, C. y Vohs, K. D. (2001). Bad is stronger than good. Review of General Psychology, 5, 323-370.

Bilbao, M. A. (2009). Creencias sociales y bienestar: valores, creencias básicas, impacto de los hechos vitales y crecimiento psicológico. (Tesis doctoral). Universidad del País Vasco, España.

Bilbao, M. A., Páez, D., da Costa, S. y Martínez-Zelaya, G. (2013). Cambio en creencias básicas y crecimiento post estrés: un estudio transversal sobre el fuerte impacto de los hechos positivos sobre el bienestar. Terapia Psicológica. 31(1), 127-139.

Bonanno, G. A. (2004). Loss, Trauma and human resilience: Have we underestimated the human capacity to the after extremely aversive events? American Psychologist, 59(1), 20-28.

Brofenbrenner, U. (1979). The ecology of human development: experiments by nature and desing. Cambridge: Harvard University Press.

Consedine, N., Magai, C. y Krivoshekova, Y. (2005). Sex and age cohort differences in patterns of socioemotional functioning in older adults and their links to physical resilience. Ageing International, 30(3), 209-244.

Corsini, S. (2004). Everyday emotional events and basic belie$f_{s}$. (Tesis doctoral). University of Louvain, Bélgica.

Csikszentmihalyi, M. (2007). Aprender a fluir. España: Kairós.

Cuadra, H. y Florenzano, R. (2003). El bienestar subjetivo: hacia una psicología positiva. Revista de Psicología de la Universidad de Chile, XII(1), 83-96.

Diener, E. (2006). Guidelines for national indicators of subjective well-being and ill-being. Applied Research in Quality of Life. The Netherlands:Springer-ISQOLS, 1, 151-157.

Diener, E. (2009). Assesing well-being: Progress and opportunities. En E. Diener (Ed.), Assessing well-being. The collected works of Ed Diener (pp. 25-65). Nueva York: Springer (Social Indicators Research Series, 39).
Diener, E. y Lucas, R. E. (2000). Subjective Emocional WellBeing. En M. Lewis y J. M. Haviland-Jones (Eds.), Handbook of emotions. New York: The Guilford.

Diener, E., Suh, E. M., Lucas, R. E. y Smith, H. L. (1999). Subjective well-being. Three decades of progress. Psychological Bulletin, 125(2), 276-302.

Fredrickson, B. L. (2009). Positivity. New York: Crown Publish.

Gaxiola, R. J. C., Frías, A. M., Hurtado A. M. F., Salcido, N. L. C. y Figueroa, F. M. (2011). Validación del inventario de resiliencia (IRES) en una población del noroeste de México. Enseñanza e Investigación en Psicología, 16(1), 73-83.

González Arratia, L. F. N. I. (2011). Resiliencia y personalidad en niños. Cómo desarrollarse en tiempos de crisis. México: Universidad Autónoma del Estado de México.

González Arratia, L. F. N. I., Amado, V. L. y Valdez, M. J. L. (2010, abril). Resiliencia y fortalezas personales en adolescentes. Sesión de Cartel presentada en el xxxviI Congreso del Consejo Nacional para la enseñanza e Investigación en Psicología (CNEIP). Chihuahua, México.

González Arratia, L. F. N. I. y Valdez, M. J. L. (2012). Análisis factorial confirmatorio del cuestionario de resiliencia en una muestra de niños. En R. Díaz Loving, A. S. Rivera e I. Reyes Lagunes (Eds.), La psicología social en México (pp. 676-681). México: UNAM.

Grotberg, E. (1995). The Internacional Resilience Proyect: Promoting Resilience in Children. ERIC: ED.383424, USA.

Grotberg, E. (2006). La resiliencia en el mundo de hoy. Barcelona: Gedisa.

Helgeson, V. S., Reynolds, K. A. y Tomich, P. L. (2006). A metaanalytic review of benefit finding and growth. Journal of Consulting and Clinical Psychology, 74(5), 797-816.

Keneally P. (1993). Hardiness, self-care practices and perceived health status in older adults. Journal of Advanced Nursing, 18(7), 1085-1094.

Landero, H. R. y González, R. M. (2006). Estadística con SPSS y metodología de la investigación. México: Trillas.

Larson, R. (1989). Is feeling "in control” related to happiness in daily life? Psychological Reports, 64, 775-784.

Levin, J. (1979). Fundamentos de estadística en la investigación social. México: Harla.

Masten, A. S. (2011). Ordinary Magic. Resilience processes in development. American Psychologist, 56, 227-238.

Michalos, A. (2007). Education, happiness and well-being. Conferencia internacional. Is happiness measurable and what do those measures mean for public policy? Roma 2-3 de abril 2007. Universidad Tor Vergata (Roma) organizada por Joint Reseach Centre of the European Commission (OECD) Centre for economic and international studies y el Banco de Italia. 
Myers, D. G. y Diener, E. (1995). Who is happy? Psychological Science, 6, 10-19. (reprinted in Annual Editions: Social Psychology 97/98; digested in Frontier Issues in Economic Thought: Vol. 3, Human Well-Being and Economic Goals, ed. Neva Goodwin).

Nygren B, Jonsen A, Gustafson Y, Norberg, A. y Lundman B. (2005). Resilience, sense of coherence, purpose in life and self-transcendence in relation to perceived physical and mental health among the oldest old. Aging \& Mental Health, 9(4), 354-362.

Omar, A. (2008). Bienestar subjetivo y perspectivas de futuro como predictores de resiliencia en adolescentes. En J. M. Moysen (Coord.), El claroscuro de la vida juvenil (pp. 5-22). México: Editorial Artes Gráficas.

Omar, A., Paris, L., Uribe, D. H., Ameida da Silva, S. H. y Aguilar de Souza, M. (2011). Un modelo explicativo de resiliencia en jóvenes y adolescentes. Psicología Em Estudo, 16(2), 269-277.

Páez, D., Bilbao, M. y Javaloy, F. (2009). Del trauma a la felicidad. En M. M. Casullo (Ed.), Prácticas en psicología positiva (pp. 159-202). Argentina: Lugar Editorial.

Páez, D., Campos, M. y Bilbao, M. A. (2008). Del trauma a la felicidad: pautas para la intervención. En Vázquez y Hervás (Eds.), Psicología positiva aplicada. Bilbao, España: Editorial Desclée de Brouwer.

Palomar, L. J. y Victorio, E. A. (2012). Impacto del efecto negativo sobre la resiliencia y los factores de protección que la promueven. En L. J. Palomar y R. J. Gaxiola (2012). Estudios de resiliencia en América Latina Vol. 1. Cap. 6, pp. 83-94.

Park, C. L. (1998). Stress-related growth and thriving through coping: the roles of personality and cognitive processes. Journal of Social Issues, 54(2), 267-277.

Rodríguez, M., Pereyra, M. Jofré, M., de Bortoli, M. y Labiano, L. M. (2009). Propiedades psicométricas de la escala de resiliencia versión argentina. Laboratorio de Evaluación Psicológica y Educativa, 9, 77-88.

Ruiz, P. G. y López, M. A. (2012). Resiliencia psicológica y dolor crónico. Escritos de Psicología, 5(2), 1-11.

Saavedra, G. E. y Villalta, P. M. (2008). Medición de las características resilientes, un estudio comparativo en personas entre 15 y 65 años. Liberabit, 14, 31-40.

Salgado, C. (2006). Felicidad en estudiantes de universidades nacionales de Perú, Bolivia, Paraguay y Argentina. Revista Cultura de la Universidad de San Martín de Porres, 20, 531-553.
Salgado, L. A. (2009). Felicidad, resiliencia y optimismo en estudiantes de colegios nacionales de la ciudad de Lima. Liberabit. Revista de Psicología, 15(2) 133-141.

Seligman, M., Steen, T. A., Park, N. y Peterson, C. (2005). Positive psychology progress: empirical validation of interventions. American Psychologist, 60, 416-421.

Such, E., Diener, E. y Fujita, F. (1996). Events and subjective well-being: only recent events Matter. Journal of Personality and Social Psychology, 70(5), 1091-1102.

Tennen, H. H. y Affleck, G. (2005). Benefit-finding and benefit-reminding. En C. R. Snyder y S. J. López (Eds.), Handbook of positive psychology (pp. 584-598). Oxford: Oxford University Press.

Toribio, P. L., González Arratia, L. F. N. I., Valdez, M. J. L. González, E. S. y Oudhof, B. H. (2012). Validación de la escala de felicidad de Alarcón para adolescentes mexicanos. Psicología Iberoamericana, 20(1), 71-79.

Ungar, M. (2005). Handbook for working with children and youth: pathways to resilience across cultures and contexts sage publications. Thousand Oaks California: Sage $\mathrm{Pu}-$ blications.

Vargas, M. J. E. y Villavicencio, E. J. M. (2011). Niveles de resiliencia en pacientes recientemente amputados: consejos para fortalecerla. Centro Regional de Investigación en Psicología, 5(1), 13-20. Recuperado de http://www. conductitlan.net

Vázquez, C. y Hervás, G. (2008). La ciencia del bienestar. Fundamentos de una psicología positiva. Madrid, España: Alianza.

Veenhoven, R. (2004). El estudio de la satisfacción con la vida. Intervención Psicosocial, 3, 87-116.

Veronese, G., Castigliani, M., Tombolani, M. y Said, M. (2012). My happiness is the refugee camp, my future Palestina: optimims, lifee satisfaction and perceived happiness in a group of Palestian children. Scan J. Caring Sci., 26, 467-473.

Zoellner, T. y Maercker, A. (2006). Posttraumatic growth in clinical psychology. A critical review and introduction of a two component model. Clinical Psychology Review, 26, 626-653. 